\title{
Hypernuclear matter in the Brueckner-Hartree-Fock approximation
}

\author{
H.-J. Schulze ${ }^{a}$, A. Lejeune ${ }^{a}$, J. Cugnon ${ }^{a}$, M. Baldo ${ }^{b}$, U. Lombardo ${ }^{b}$ \\ a Université de Liège, Institut de Physique B5, B-4000 Liège 1, Belgium \\ b Dipartimento di Fisica. Università di Catania. Corso Italia, 57, I-95129 Catania, Italy \\ Received 25 April 1995 \\ Editor: G.F. Bertsch
}

\begin{abstract}
We present a self-consistent calculation of nucleon and hyperon single-particle potentials for a system of nucleons and $\Lambda$ hyperons of nonzero densities $\rho_{N}, \rho_{\Lambda}$ using the Brueckner-Hartree-Fock approximation scheme. Fixing the nucleon density at $\rho_{N}=\rho_{0}$ we find a minimum of the total binding energy per baryon at a finite ratio $\rho_{\Lambda} / \rho_{N} \approx 0.1$ and a corresponding gain of binding energy per baryon of about $0.4 \mathrm{MeV}$.
\end{abstract}

PACS: 21.65.+f; 21.80.+a

The availability of reliable nucleon-hyperon potentials $[1,2]$ has led to the possibility of investigating many-body systems consisting of nucleons and hyperons in a quantitative manner. The motivation for such investigations arises from the interest in constructing an effective, density dependent nucleon-hyperon potential for the use in shell-model hypernuclei calculations [3-7], from the search for production of multistrange clusters in relativistic heavy ion collisions [8], and from speculations concerning "strangelets", i.e. strongly bound systems of strange matter which may even be stable against weak decay [9]. A more exotic application concerns the structure of neutron stars, which are thought to consist in the core region of basically hyperonic matter under extreme densities [10].

Up to date, these investigations of hypernuclear matter have been carried out either in relativistic mean-field (RMF) calculations [4-6], or within the framework of an extension of Brueckner-HartreeFock (BHF) theory for a mixture of nuclear matter and hyperonic matter [3]. These latter works, however, proceed in a non self-consistent way, i.e. by concentrating on the action of a medium of nucleons on an isolated hyperon, and neglecting the effects of the thus calculated hyperon self-energy on the nucleons in the case of a finite density of hyperons in the medium. It is the purpose of this article to provide such a self-consistent calculation.

Our calculations are based on the Paris nucleonnucleon [11] and the Nijmegen soft-core nucleonhyperon [1] potentials. This choice constitutes the natural extension of our previous work on nuclear matter using the Paris potential [12], by the introduction of the most elaborated version of the nucleon-hyperon interaction available up to now. Unfortunately we are not aware of reliable hyperon-hyperon, in particular $\Lambda-\Lambda$ potentials, so that, for the time being, we neglect 
the hyperon-hyperon interaction in our calculation and are thus somehow restricted to small lambda densities. Our main interest lies in the possible application to hypernuclei and heavy-ion collisions, in which this restriction might be justified.

Let us establish our notation. Both the Paris nucleon-nucleon and the Nijmegen nucleon-hyperon potentials are given in the common general form

$$
\begin{aligned}
V_{12} & =V_{C}(r)+\frac{1}{2}[\Delta \phi(r)+\phi(r) \Delta] \\
& +V_{S S}(r) \sigma_{1} \cdot \sigma_{2}+V_{L S}(r) L \cdot S \\
& +V_{Q}(r) \frac{1}{2}\left[\left(\sigma_{1} \cdot L\right)\left(\sigma_{2} \cdot L\right)+\left(\sigma_{2} \cdot L\right)\left(\sigma_{1} \cdot L\right)\right] \\
& \left.+V_{T}(r) 3\left(\sigma_{1} \cdot \hat{\boldsymbol{r}}\right)\left(\sigma_{2} \cdot \hat{\boldsymbol{r}}\right)-\left(\sigma_{1} \cdot \sigma_{2}\right)\right],
\end{aligned}
$$

i.e. containing a central and momentum dependent component as well as spin-spin, linear and quadratic spin-orbit, and tensor parts. This fact allows a reliable numerical treatment on equal footing.

Using these potentials we have to solve the BetheGoldstone $[13,14]$ equation in the nucleon-nucleon and nucleon-hyperon channel. We write down the equation for the latter case:

$$
\begin{aligned}
& u_{Y Y^{\prime}, L L^{\prime}}(k, r)=j_{L}(k r) \delta_{Y Y^{\prime}} \delta_{L L^{\prime}} \\
& +4 \pi \int_{0}^{\infty} d r^{\prime} r^{2} D_{Y Y^{\prime}, L^{\prime}}\left(r, r^{\prime}\right) \\
& \quad \times \sum_{Y^{\prime \prime}, L^{\prime \prime}} V_{Y^{\prime} Y^{\prime \prime}, L^{\prime} L^{\prime \prime}}\left(r^{\prime}\right) u_{Y Y^{\prime \prime}, L L^{\prime \prime}}\left(k, r^{\prime}\right)
\end{aligned}
$$

with

$$
\begin{array}{r}
D_{Y Y^{\prime}, L^{\prime}}\left(r, r^{\prime}\right)=-\frac{1}{2 \pi^{2}} \int_{0}^{\infty} d k^{\prime} k^{2} \\
\times \frac{j_{L^{\prime}}\left(k^{\prime} r\right) j_{L^{\prime}}\left(k^{\prime} r^{\prime}\right) f_{Y^{\prime}}\left(k^{\prime}\right)}{E_{Y}(k)-E_{Y^{\prime}}\left(k^{\prime}\right)}
\end{array}
$$

and

$$
\begin{aligned}
& E_{Y}(k)=\frac{k_{N}^{2}}{2 M_{N}}+\frac{k_{Y}^{2}}{2 M_{Y}}+U_{N}\left(k_{N}\right)+U_{\Lambda}\left(k_{Y}\right) \\
& \quad+M_{N}+M_{Y} .
\end{aligned}
$$

Here $k$ and $k^{\prime}$ denote the relative momenta of the initial $N Y$ and the intermediate $N Y^{\prime}$ state, $E_{Y}(k)$ and $E_{Y^{\prime}}\left(k^{\prime}\right)$ are the corresponding energies, and $f_{Y^{\prime}}\left(k^{\prime}\right)$ denotes the angle-averaged Pauli operator in the intermediate states.
In these expression $Y, Y^{\prime}, Y^{\prime \prime}=\Lambda, \Sigma$ account for the possibility of $N \Lambda \leftrightarrow N \Sigma$ mixing through the strong interaction, and the equation has to be solved for a set of states with definite quantum numbers $T, S, J$, which have not been indicated explicitly. The BetheGoldstone equation has thus a $2 \times 2$ matrix structure due the coupling between nucleon-lambda and nucleon-sigma states and a $4 \times 4$ structure when the mixing of angular momentum states through the tensor potential applies.

The solutions of the Bethe-Goldstone equation determine the diagonal $G$-matrix elements

$$
\begin{aligned}
& \left\langle k_{N} k_{Y}\left|G_{Y, L}(T, S, J)\right| k_{N} k_{Y}\right\rangle=4 \pi \int_{0}^{\infty} d r r^{2} j_{L}(k r) \\
& \times \sum_{Y^{\prime}, L^{\prime}} V_{Y Y^{\prime}, L L^{\prime}}(r) u_{Y Y^{\prime}, L L^{\prime}}(k, r),
\end{aligned}
$$

and the single-particle polentials are then given by

$$
\begin{gathered}
U_{A}^{(B)}\left(k_{A}\right)=\sum_{T, S, J, L} \frac{(2 T+1)(2 J+1)}{\left(2 t_{A}+1\right)\left(2 s_{A}+1\right)} \frac{1}{2 \pi^{2}} \\
\times \int_{0}^{k_{F}^{(B)}} d k_{B} k_{B}^{2}\left\langle k_{A} k_{B}\left|G_{A, L}(T, S, J)\right| k_{A} k_{B}\right\rangle,
\end{gathered}
$$

where the notation $U_{A}^{(B)}$ denotes the single-particle potential of particle $A$ due to the interaction with particles $B$ in the medium. Carrying out the calculation for the four combinations $A=N, \Lambda ; B=N, \Lambda$, we obtain finally the total single-particle potentials of nucleons and lambdas as

$$
\begin{aligned}
& U_{N}\left(k_{N}\right)=U_{N}^{(N)}\left(k_{N}\right)+U_{N}^{(\Lambda)}\left(k_{N}\right), \\
& U_{\Lambda}\left(k_{\Lambda}\right)=U_{\Lambda}^{(N)}\left(k_{\Lambda}\right)+U_{\Lambda}^{(\Lambda)}\left(k_{\Lambda}\right) .
\end{aligned}
$$

(Presently we set $U_{\Lambda}^{(\Lambda)}=0$ due to the neglect of the lambda-lambda interaction). We are interested in the total binding energy per baryon. In the BHF approximation the binding energy $B$ and the baryon number $A$ per unit volume are given by 


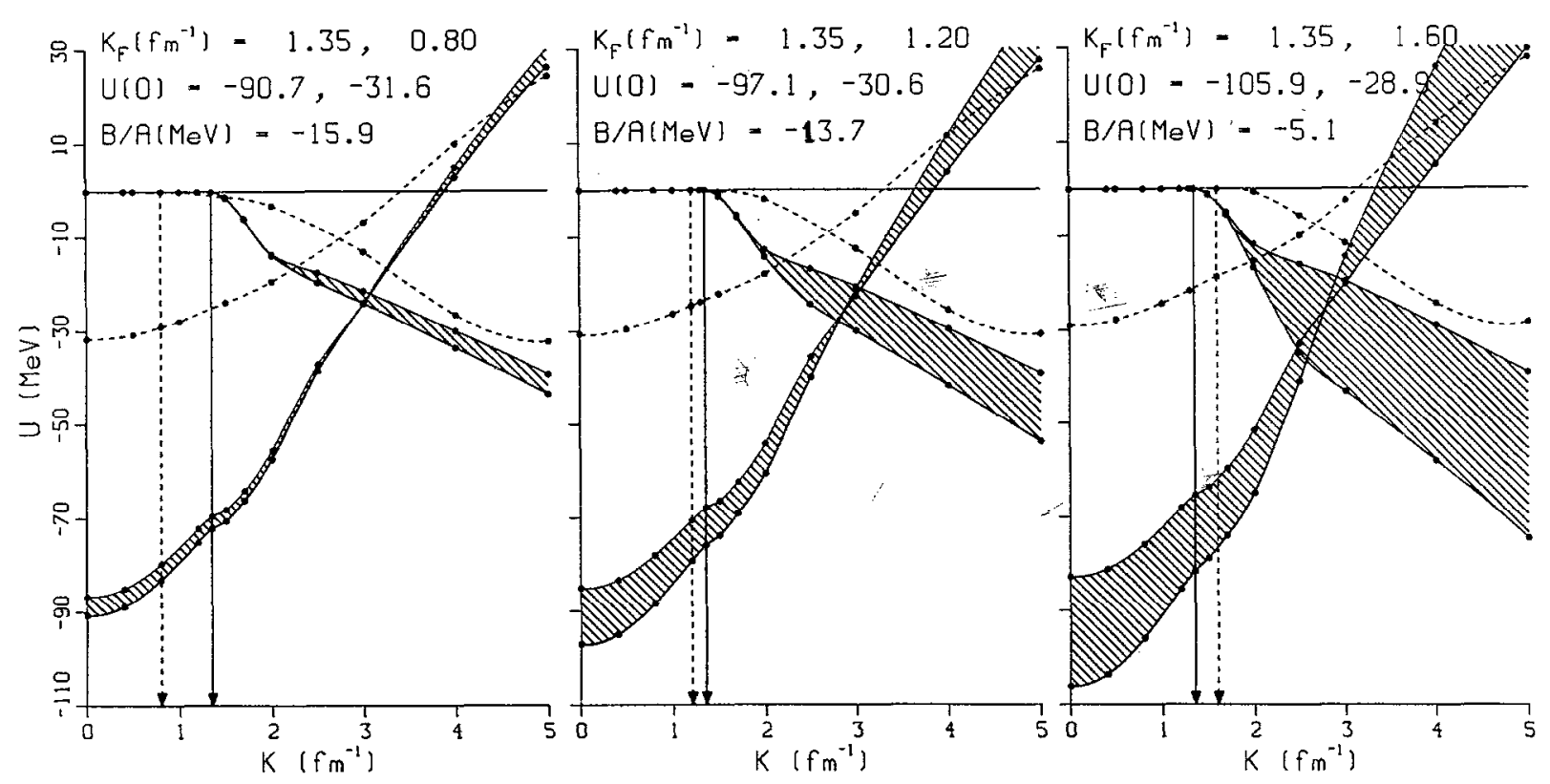

Fig. 1. The nucleon and lambda single-particle potentials (real and imaginary parts) for three values of $k_{F}^{(\Lambda)}=0.8,1.2,1.6 \mathrm{fm}{ }^{-1}$ at fixed $k_{F}^{(N)}=1.35 \mathrm{fm}^{-1}$. The dashed curves represent $U_{\Lambda}^{(N)}$, and the full curves $U_{N}$ (lower curve) and $U_{N}^{(N)}$ (upper curve). The shaded areas thus represent $U_{N}^{(\Lambda)}$. The real parts correspond to the increasing functions whereas the imaginary parts are given by the curves with a negative slope. The arrows denote the positions of the Fermi momenta.

$$
\begin{aligned}
B & =\frac{4 \pi}{(2 \pi)^{3}}\left[4 \int_{0}^{k_{F}^{(N)}} d k k^{2}\left(\frac{k^{2}}{2 M_{N}}+\frac{1}{2} U_{N}(k)\right)\right. \\
& \left.+2 \int_{0}^{k_{F}^{(\Lambda)}} d k k^{2}\left(\frac{k^{2}}{2 M_{\Lambda}}+\frac{1}{2} U_{\Lambda}(k)\right)\right]
\end{aligned}
$$

and

$$
A=\frac{1}{3 \pi^{2}}\left(2 k_{F}^{(N)^{3}}+k_{F}^{(\Lambda)^{3}}\right) .
$$

We come now to the presentation of our results. Our code has been tested by first carrying out ordinary nuclear matter calculations, i.e. for vanishing hyperon densities. The results are in good agreement with previous BHF calculations using the Paris potential [15].

We then proceed to the simultaneous, self-consistent determination of nucleon and lambda single-particle potentials in an environment of nucleon density $\rho_{N}$ and non-zero hyperon density $\rho_{\Lambda}$. In this article we will restrict our investigation to a variation of the lambda density for fixed nucleon density $\rho_{N}=\rho_{0}=0.17 \mathrm{fm}^{-3}$, which is relevant for the situation in heavy hypernuclei. As an illustration we show in Fig. 1 three sets of single-particle potentials for fixed $k_{F}^{(N)}=1.35 \mathrm{fm}^{-1}$ and different values of $k_{F}^{(\Lambda)}=0.8,1.2,1.6 \mathrm{fm}^{-1}$, corresponding to density ratios $\rho_{\Lambda} / \rho_{N}=0.10,0.35,0.80$.

The $U_{N}^{(N)}$ part of the nucleon mean field turns out to be very close to the mean field in undisturbed nuclear matter. There is only a slight reduction of the potential due to the self-consistency requirement: the $G$-matrix in the $N N$ channel is just changed by the addition of the $U_{N}^{(\Lambda)}$ contribution in the denominator. Since this contribution is slowly varying with momentum, the denominator changes very little and the resulting change of $U_{N}^{(N)}$ is quite small, as can be seen by comparing the corresponding curves at different lambda densities in Fig. 1. Due to the absence of a $\Lambda \Lambda$ interaction the potentials $U_{\Lambda}=U_{\Lambda}^{(N)}$ are basically unchanged on varying the lambda density. Only a slight reduction due to the increased lambda Pauli blocking in the intermediate states is to be seen. When the lambda density is varied, the dominant effect is thus the substantial enhancement of the total nucleon potential due to 
Table 1

The contributions (in MeV) of various partial waves to the single-particle potential $U_{\Lambda}\left(k_{\Lambda}=0\right)$ at $k_{F}^{(N)}=1.35 \mathrm{fm}^{-1}$ and $k_{F}^{(\Lambda)}=0$

\begin{tabular}{|c|c|c|c|c|c|c|c|c|}
\hline \multirow{3}{*}{$\begin{array}{l}\text { State } \\
{ }^{1} S_{0} \\
{ }^{3} P_{0}\end{array}$} & \multicolumn{2}{|c|}{$(\Lambda \Lambda)$} & \multicolumn{2}{|c|}{$(\Lambda \Sigma)$} & \multicolumn{2}{|c|}{$(\Lambda \Lambda)+(\Lambda \Sigma)$} & & \\
\hline & -12.7 & $(-13.8)$ & -2.3 & $(-2.2)$ & -15.1 & $(-16.0)$ & & \\
\hline & 0.4 & & -0.1 & & 0.2 & $(0.3)$ & & \\
\hline${ }^{3} S S_{1}$ & 17.3 & (15.6) & -10.2 & $(-8.3)$ & 7.0 & (7.3) & & \\
\hline${ }^{3} S D_{1}$ & -2.1 & $(-2.2)$ & -20.8 & $(-19.9)$ & -22.9 & $(-22.1)$ & & \\
\hline${ }^{3} D D_{1}$ & -0.06 & & -0.02 & & -0.08 & & & \\
\hline${ }^{3} D S_{1}$ & -0.00 & & -0.02 & & -0.02 & & -15.9 & $(-14.8)$ \\
\hline${ }^{1} P_{1}$ & 2.0 & & -0.4 & & 1.5 & (1.6) & & \\
\hline${ }^{3} P_{1}$ & 2.2 & & -0.3 & & 1.9 & $(1.7)$ & & \\
\hline${ }^{3} P_{2}$ & -3.0 & & -0.4 & & -3.3 & & & \\
\hline${ }^{3} P_{F_{2}}$ & -0.0 & & -0.8 & & -0.8 & & & \\
\hline${ }^{3} F F_{2}$ & -0.01 & & -0.00 & & -0.01 & & & \\
\hline${ }^{3} \mathrm{FP}_{2}$ & -0.00 & & -0.00 & & -0.00 & & -4.1 & $(-3.8)$ \\
\hline${ }^{1} D_{2}$ & -0.4 & & -0.01 & & -0.4 & & & \\
\hline${ }^{3} D_{2}$ & -0.4 & & -0.06 & & -0.4 & & & \\
\hline sum & & & & & -32.3 & $(-30.8)$ & & \\
\hline
\end{tabular}

Where available, the numbers in brackets show the comparable results of Ref. [3]. The numbers in the last column give the summed contributions for the corresponding coupled partial waves.

the additional attraction of the nucleons by the hyperons in the medium, as given by $U_{N}^{(\Lambda)}$. Even neglecting the $\Lambda \Lambda$ interaction, this nucleon-hyperon attraction provides significant extra potential energy to the system. Let us also mention that the effective mass of the lambda has a typical value $M_{\Lambda}^{*} / M_{\Lambda} \approx 0.81$, whereas the nucleon effective mass decreases slightly with increasing lambda density.

The imaginary parts of the nucleon and lambda mean field are also displayed in Fig. 1. One can observe that the nucleon-hyperon interaction brings a stronger relative change to this quantity than to the real part of the potential, especially at large lambda densities. We recall that these imaginary parts arise from collisions and, for the hyperon, do not include weak interactions.

We come back to the discussion of the binding energy a bit later, but would first like to compare quantitatively our results for the limiting case $k_{F}^{(\Lambda)}=0$, i.e. for an isolated hyperon in a nucleonic environment, with those of Ref. [3]: In both calculations the continuous BHF scheme together with the Nijmegen soft-core potentials and partial waves up to $L=2$ are used. The main differences are the nucleon singleparticle potentials $U_{N}^{(N)}$ (in our case BHF with Paris potential, whereas the choice of Ref. [3] is not explicitly known), and the sigma potentials $U_{\Sigma}$ employed in the intermediate states (we approximate $U_{\Sigma}(k) \approx$ $U_{\Lambda}(k)$, whereas the explicitly calculated $U_{\Sigma}(k)$ is used in Ref. [3]). The latter difference does play a minor role, as the associated mass difference $M_{\Sigma}$ $M_{\Lambda} \approx 77 \mathrm{MeV}$ is dominating in the energy denominator of the Bethe-Goldstone equation. We therefore find a fairly good agreement between both calculations: our lambda mean field is $32.3 \mathrm{MeV}$ deep, whereas the value of Ref. [3] is $30.8 \mathrm{MeV}$. For a closer comparison, we list in Table 1 the contributions of the various partial waves to the lambda well depth $U_{\Lambda}(0)$. Also here we find good agreement between both works.

It is gratifying to see that the lambda mean field obtained by the BHF method (with the so-called continuous choice) plus the Nijmegen potential agrees quite well with the lambda average potential extracted from hypernuclei phenomenology [16], which is about $30 \mathrm{MeV}$ deep. One has however to remind that higher order diagrams in the Bethe-Goldstone expan- 




Fig. 2. Total binding energy (full curve, scale on the left) and lambda Fermi energy (dashed curve, scale on the right) as functions of $k_{F}^{(\Lambda)}$ for fixed $k_{F}^{(N)}=1.35 \mathrm{fm}^{-1}$.

sion are likely to change the calculated value by as much as $10 \%$ [17] and that the relationship between the lambda interaction average potentials in nuclei and in infinite matter may not be a simple equality.

In order to assess the stability of the hypernuclear matter with respect to strong interaction, we then display in Fig. 2 the total binding energy per baryon as given by Eqs. (8), (9), as well as the Fermi energy of the lambda, $e_{F}^{(\Lambda)}=k_{F}^{(\Lambda)^{2}} / 2 M_{\Lambda}+U_{\Lambda}\left(k_{F}^{(\Lambda)}\right)$, for fixed nucleon density $k_{F}^{(N)}=1.35 \mathrm{fm}^{-1}$ and varying lambda density. We find that the binding energy per baryon assumes a minimum at $k_{F}^{(\Lambda)} \approx 0.8 \mathrm{fm}^{-1}$, i.e. $\rho_{\Lambda} / \rho_{N} \approx 0.1$. The gain in binding energy per particle at this minimum compared to pure nucleonic matter is about $0.4 \mathrm{MeV}$. This is a novel result in the context of a BHF calculation, and it agrees quite well with similar findings in RMF calculations [4,5]. Let us mention that this minimum does not imply stability against weak interaction as the mass difference is not included in the quantity $B / A$. It however emphasizes the stability of hypernuclei with many lambdas against strong interaction. The lambda Fermi energy vanishes for $k_{F}^{(\Lambda)} \approx 1.2 \mathrm{fm}^{-1}$. This corresponds to the impossibility of adding a further lambda to the matter with the corresponding $\rho_{\Lambda}$ and $\rho_{N}$ values, as this particle cannot be bound any more. If a local density approximation is valid, the limiting value of $\rho_{\Lambda} / \rho_{N}$, i.e. the ratio of the number of lambdas to the number of nucleons, in a hypernucleus lies around 0.35 . (In the RMF calculation of Ref. [6] a ratio $\rho_{\Lambda} / \rho_{N} \approx 1 / 3$ was found in heavy hypernuclei). Of course, this conclusion assumes that the nucleonic matter inside the hypernucleus is not distorted too much by the presence of the lambdas.

In conclusion, we have presented the first selfconsistent BHF calculation of nucleon and hyperon single-particle properties in a uniform infinite matter made of neutrons and protons in equal proportions and of lambdas. Two important results are obtained. First, the lambda mean field in basically nuclear matter $(\approx 32 \mathrm{MeV})$ is in good agreement with the phenomenological value extracted from hypernuclei systematics $(\approx 30 \mathrm{MeV})$ [16] and with the result of the comparable calculation of Ref. [3] $(\approx 31 \mathrm{MeV})$. Second, the binding energy per baryon of the matter has a shallow minimum for $\rho_{\Lambda} / \rho_{N} \approx 0.1$, and there is a critical density ratio $\rho_{\Lambda} / \rho_{N} \approx 0.35$, beyond which an additional hyperon will no longer be bound.

Our calculation presently neglects the hyperonhyperon interaction because of its great complexity (coupled channels) and the lack of experimental constraints on its possible form. Note also that the presence of a substantial $\Lambda$ density may imply also a finite $\Xi$ density, due to the Pauli-blocking of the decay $\Xi N \rightarrow \Lambda \Lambda[6,18]$. One can therefore only speculate at the moment that the inclusion of the presumably globally attractive hyperon-hyperon interaction will increase the binding energy of the system even more and lead to a minimum at a larger strangeness content of the matter. On the other hand these effects might not be too large, since for hyperon densities larger than the ones investigated here, i.e. larger than $0.4 \rho_{0}$, the strong repulsive parts of the $\Lambda N$ and $\Lambda \Lambda$ interactions start to play an important role. An estimate of the effect of including a $\Lambda \Lambda$ interaction as well as a detailed account of our numerical procedure will be given in an extended publication.

We would like to thank T. Rijken and J. de Swart for providing us with their nucleon-hyperon potential code and for an interesting discussion. This work was supported by EC contract ERBCHRX-CT92-0075. 


\section{References}

[1] P. Maessen, Th. Rijken and J. de Swart, Phys. Rev. C 40 (1989) 2226.

[2] A. Reuber, K. Holinde and J. Speth, Nucl. Phys. A 570 (1994) 543.

[3] Y. Yamamato and H. Bando, Prog. Theor. Phys. 83 (1990) 254.

[4] J. Mareš and J. Žofka, Z. Phys. A 333 (1989) 209; A 345 (1993) 47.

[5] M. Rufa et al., Phys. Rev. C 42 (1990) 2469;

J. Schaffner, C. Greiner and H. Stöcker, Phys. Rev. C 46 (1992) 322.

[6] J. Schaffner et al., Ann. Phys. (N.Y.) 235 (1994) 35.

[7] J. Hao et al., Phys. Rev. Lett. 71 (1993) 1498.

[8] C. Greiner, D.-H. Rischke, H. Stöcker and P. Koch, Phys. Rev. D 38 (1988) 2797;

A.J. Baltz et al., Phys. Lett. B 325 (1994) 7.

[9] E. Witten, Phys. Rev. D 30 (1984) 2379; G.R. Young, Nucl. Phys. A 544 (1992) 391c.

[10] A.G.W. Cameron, Astrophys. J. 130 (1959) 884; V.R. Pandharipande, Nucl. Phys. A 178 (1971) 123;
H.A. Bethe and M.B. Johnson, Nucl. Phys. A 230 (1974) 1;

N.K. Glendenning, Nucl. Phys. A 493 (1989) 521.

[11] M. Lacombe et al., Phys. Rev. C 21 (1980) 861.

[12] A. Lejeune, P. Grangé, M. Martzolff and J. Cugnon, Nucl. Phys. A 453 (1986) 189.

[13] K.A. Brueckner and J.L. Gammel, Phys. Rev. 109 (1958) 1023.

[14] J.-P. Jeukenne, A. Lejeune and C. Mahaux, Phys. Rep. C 25 (1976) 83.

[15] B.D. Day and R.B. Wiringa, Phys. Rev. C 32 (1985) 1057; T.T.S. Kuo, Z.Y. Ma and R. Vinh Mau, Phys. Rev. C 33 (1986) 717;

M. Baldo et al., Phys. Rev. C 43 (1991) 2605.

[16] H. Feshbach, Nucl. Phys. A 507 (1990) 219c;

C.B. Dover and A. Gal, Prog. Part. Nucl. Phys. 12 (1984) 171;

D.J. Millener, C.B. Dover and A. Gal, Phys. Rev. C 38 (1988) 2700;

C.B. Dover, D.J. Millener, A. Gal and D.H. Davis, Phys. Rev. C 44 (1991) 1905.

[17] J. Rożynek and J. Dąbrowski, Phys. Rev. C 20 (1979) 1612.

[18] C.B. Dover and A. Gal, Nucl. Phys. A 560 (1993) 559c. 